\title{
Minimizing Oblique Errors for Robust Estimating
}

\author{
DIARMUID O'DRISCOLL, DONALD E. RAMIREZ,
} AND REBECCA SCHMITZ

\begin{abstract}
The slope of the best fit line from minimizing the sum of the squared oblique errors is shown to be the root of a polynomial of degree four. We introduce a median estimator for the slope and, using a case study, we show that the median estimator is robust.
\end{abstract}

\section{INTRODUCTION}

With ordinary least squares (OLS) regression, we have data

$$
\left\{\left(x_{1}, Y_{1} \mid X=x_{1}\right), \ldots,\left(x_{n}, Y_{n} \mid X=x_{n}\right)\right\}
$$

and we minimize the sum of the squared vertical errors to find the best-fit line $y=h(x)=\beta_{0}+\beta_{1} x$. With OLS it is assumed that the independent or causal variable is measured without error.

J. L. Gill [2] states that "some regression prediction or estimation must be made in a direction opposite to the natural causality of one variable by another." This is found from the inverse function $h^{-1}\left(y_{0}\right)=x_{0}=y_{0} / \beta_{1}-\beta_{0} / \beta_{1}$. He adds "Geometric mean regression could be more valid than either direct or inverse regression if both variables are subject to substantial measurement error."

For inverse prediction we will want both $h(x)$ and $h^{-1}(y)$ to model the data. To accomplish this, we try to determine a fit so that the squared vertical and the squared horizontal errors will both be small. The vertical errors are the squared distances from $(x, y)$ to $(x, h(x))$ and the horizontal errors are the squared distances from $(x, y)$ to $\left(h^{-1}(y), y\right)$. As a compromise, we will consider the errors at the median or midpoint to the predicted vertical and predicted horizontal values. All of the estimated regression models we consider (including the geometric mean and perpendicular methods) are contained in the parametrization (with $0 \leq \lambda \leq 1$ ) of the line from $(x, h(x))$ 
to $\left(h^{-1}(y), y\right)$. For the squared vertical errors, set $\lambda=1$ and correspondingly, for the horizontal errors, set $\lambda=0$. Our Maple codes and the data set for our case study can be found here:

$$
\text { people.virginia.edu/ der/pdf/oblique_errors }
$$

Our paper first introduces the Oblique Error Method in Section 2. In Section 3, we show how the Geometric Mean and Perpendicular Methods are included in our parametrization. In Section 4, we include a weighted regression procedure and Section 5 contains a small case study showing the robustness of the proposed median slope estimator.

\section{Minimizing Squared Oblique Errors}

From the data point $\left(x_{i}, y_{i}\right)$ to the fitted line $y=h(x)=\beta_{0}+\beta_{1} x$ the vertical length is $a_{i}=\left|y_{i}-\beta_{0}-\beta_{1} x_{i}\right|$, the horizontal length is $b_{i}=\left|x_{i}-\left(y_{i}-\beta_{0}\right) / \beta_{1}\right|=\left|\left(\beta_{1} x_{i}-y_{i}+\beta_{0}\right) / \beta_{1}\right|=\left|a_{i} / \beta_{1}\right|$ and the perpendicular length is $h_{i}=a_{i} / \sqrt{1+\beta_{i}^{2}}$. With standard notation,

$$
S_{x x}=\sum_{i=1}^{n}\left(x_{i}-\bar{x}\right)^{2}, S_{y y}=\sum_{i=1}^{n}\left(y_{i}-\bar{y}\right)^{2}, S_{x y}=\sum_{i=1}^{n}\left(x_{i}-\bar{x}\right)\left(y_{i}-\bar{y}\right)
$$

with the correlation $\rho=S_{x y} / \sqrt{S_{x x} S_{y y}}$. A basic fact is $-1 \leq \rho \leq 1$ or equivalently $0 \leq S_{x y}^{2} \leq S_{x x} S_{y y}$.

For the oblique length from $\left(x_{i}, y_{i}\right)$ to $\left(h^{-1}\left(y_{i}\right)+\lambda\left(x_{i}-h^{-1}\left(y_{i}\right)\right), y_{i}+\right.$ $\left.\lambda\left(h\left(x_{i}\right)-y_{i}\right)\right)$, the horizontal length is $(1-\lambda) b_{i}=(1-\lambda) a_{i} / \beta_{1}$ and the vertical length is $\lambda a_{i}$. Since $S S E_{h}\left(\beta_{0}, \beta_{1}, \lambda\right)=\left(\sum_{i=1}^{n} a_{i}^{2}\right) / \beta_{1}^{2}$ and $S S E_{v}\left(\beta_{0}, \beta_{1}, \lambda\right)=\sum_{i=1}^{n} a_{i}^{2}$, we have

$$
\begin{aligned}
& S S E_{o}\left(\beta_{0}, \beta_{1}, \lambda\right)=(1-\lambda)^{2} S S E_{h}+\lambda^{2} S S E_{v} \\
& =\sum_{i=1}^{n}\left\{\frac{(1-\lambda)^{2} a_{i}^{2}}{\beta_{1}^{2}}+\lambda^{2} a_{i}^{2}\right\}=\frac{(1-\lambda)^{2}+\lambda^{2} \beta_{1}^{2}}{\beta_{1}^{2}} \sum_{i=1}^{n} a_{i}^{2} .
\end{aligned}
$$

Setting $\partial S S E_{o} / \partial \beta_{0}=0$, then $\beta_{0}=\bar{y}-\beta_{1} \bar{x}$ and

$$
\begin{aligned}
\sum_{i=1}^{n} a_{i}^{2} & =\sum_{i=1}^{n}\left\{\left(y_{i}-\bar{y}\right)-\beta_{1}\left(x_{i}-\bar{x}\right)\right\}^{2} \\
& =S_{y y}-2 \beta_{1} S_{x y}+\beta_{1}^{2} S_{x x} .
\end{aligned}
$$

Hence

$$
S S E_{o}=\left((1-\lambda)^{2} \beta_{1}^{-2}+\lambda^{2}\right)\left(S_{y y}-2 \beta_{1} S_{x y}+\beta_{1}^{2} S_{x x}\right)
$$


with

$\frac{\partial S S E_{o}}{\partial \beta_{1}}=-2(1-\lambda)^{2} \beta_{1}^{-3} S_{y y}+2(1-\lambda)^{2} \beta_{1}^{-2} S_{x y}-2 \lambda^{2} S_{x y}+2 \lambda^{2} \beta_{1} S_{x x}$.

Thus the oblique estimator is a root of the fourth degree polynomial in $\beta_{1}$, namely

$$
P_{4}\left(\beta_{1}\right)=\lambda^{2} \sqrt{\frac{S_{x x}}{S_{y y}}} \beta_{1}^{4}-\lambda^{2} \rho \beta_{1}^{3}+(1-\lambda)^{2} \rho \beta_{1}-(1-\lambda)^{2} \sqrt{\frac{S_{y y}}{S_{x x}}} .
$$

We claim that $P_{4}\left(\beta_{1}\right)$ has exactly two real roots, one positive and one negative. By inspection, since the leading coefficient of $P_{4}\left(\beta_{1}\right)$ is positive and the constant coefficient is negative, $P_{4}\left(\beta_{1}\right)$ necessarily has at least one positive and one negative root. That these are the only real roots will be important in establishing the global minimum value for $S S E_{o}$.

The Complete Discrimination System $\left\{D_{1}, \ldots, D_{n}\right\}$ of Yang [4] is a set of explicit expressions that determine the number (and multiplicity) of roots of a polynomial. In the case of a fourth degree polynomial, the polynomial has exactly two real roots, each with multiplicity one, provided $D_{4}<0$; where $D_{4}=256 a_{0}^{3} a_{4}^{3}+\ldots+144 a_{0}^{2} a_{2} a_{4} a_{3}^{2}$. The expression for $D_{4}$ has 16 terms involving the five coefficients $\left\{a_{0}, \ldots, a_{4}\right\}$ of the polynomial and it is of order 6 .

For the polynomial $P_{4}\left(\beta_{1}\right)$ (with some manipulations),

$$
\begin{aligned}
D_{4}= & \lambda^{6}(1-\lambda)^{6}\left(-256+192 \rho^{2}+6 \rho^{4}+4 \rho^{6}\right) \\
& -27 \lambda^{4}(1-\lambda)^{4} \rho^{4}\left(\frac{S_{x x}}{S_{y y}}(1-\lambda)^{4}+\lambda^{4} \frac{S_{y y}}{S_{x x}}\right) .
\end{aligned}
$$

Since $|\rho| \leq 1$, it follows that $D_{4}<0$. And thus $P_{4}\left(\beta_{1}\right)$ has exactly one positive and one negative root.

Evaluating $\partial S S E_{o} / \partial \beta_{1}$ at $\beta_{1}=S_{x y} / S_{x x}$ and using the inequality $0 \leq S_{x y}^{2} \leq S_{x x} S_{y y}$ and the equality $S_{x x} S_{y y}-S_{x y}^{2}=\left(1-\rho^{2}\right) S_{x x} S_{y y}$,

$$
\begin{aligned}
\frac{\partial S S E_{o}}{\partial \beta_{1}} & =\frac{-2(1-\lambda)^{2}}{\beta_{1}^{2}}\left\{\frac{S_{y y}}{S_{x y} / S_{x x}}-S_{x y}\right\}+2 \lambda^{2}\left\{-S_{x y}+\frac{S_{x y}}{S_{x x}} S_{x x}\right\} \\
& =\frac{-2(1-\lambda)^{2}}{\beta_{1}^{2}} \frac{1}{S_{x y}} S_{x x} S_{y y}\left(1-\rho^{2}\right)
\end{aligned}
$$


which has the sign of $-S_{x y}$. Similarly evaluating $\partial S S E_{o} / \partial \beta_{1}$ at $\beta_{1}=S_{y y} / S_{x y}$

$$
\frac{\partial S S E_{o}}{\partial \beta_{1}}=2 \lambda^{2} \frac{1}{S_{x y}} S_{y y} S_{x x}\left(1-\rho^{2}\right)
$$

which has the sign of $S_{x y}$.

We use the Intermediate Value Theorem to assert that (1) If $S_{x y}>0$, then $0<S_{x y} / S_{x x} \leq \beta_{1} \leq S_{y y} / S_{x y} ;$ (2) If $S_{x y}<0$, then $S_{y y} / S_{x y} \leq \beta_{1} \leq S_{x y} / S_{x x}<0$; and (3) If $S_{x y}=0, \beta_{1}=$ $\pm\left(\left((1-\lambda)^{2} S_{y y}\right) /\left(\lambda^{2} S_{x x}\right)\right)^{1 / 4}$.

The Second Derivative Test assures that a root of $P_{4}\left(\beta_{1}\right)$ is a local minimum of $S S E_{o}$ by

$$
\begin{aligned}
\frac{\partial^{2} S S E_{o}}{\partial \beta_{1}^{2}} & =\frac{6(1-\lambda)^{2} S_{y y}}{\beta_{1}^{4}}-\frac{4(1-\lambda)^{2} S_{x y}}{\beta_{1}^{3}}+2 \lambda^{2} S_{x x} \\
& =\frac{2(1-\lambda)^{2}}{\beta_{1}^{4}}\left[3 S_{y y}-2 \beta_{1} S_{x y}\right]+2 \lambda^{2} S_{x x},
\end{aligned}
$$

with $3 S_{y y}-2 \beta_{1} S_{x y}=3 S_{y y}-2\left|\beta_{1} S_{x y}\right| \geq 3 S_{y y}-2 S_{y y}=S_{y y}>0$.

Suppose $S_{x y}>0$. Note from Equation (1) that $S S E_{o}\left(\left|\beta_{1}\right|\right)<$ $S S E_{o}\left(-\left|\beta_{1}\right|\right)$. Let $\beta_{1}^{+}$be the positive root of $P_{4}\left(\beta_{1}\right)$ and let $\beta_{1}^{-}$ be the negative root of $P_{4}\left(\beta_{1}\right)$. Then $S S E_{o}\left(\beta_{1}^{+}\right) \leq S S E_{o}\left(\left|\beta_{1}^{-}\right|\right)<$ $S S E_{o}\left(\beta_{1}^{-}\right)$. This assures that the positive root gives the global minimum for $S S E_{0}\left(\beta_{1}\right)$. A similar result holds when $S_{x y}<0$.

\section{Minimizing Squared Perpendicular and Squared Geometric Mean Errors}

The perpendicular error model dates back to Adcock [1] who introduced it as a procedure for fitting a straight line model to data with error measured in both the $x$ and $y$ directions.

For squared perpendicular errors we minimize $S S E_{p}\left(\beta_{0}, \beta_{1}\right)=$ $\sum_{i=1}^{n} a_{i}^{2} /\left(1+\beta_{1}^{2}\right)$ with solutions $\beta_{0}^{p}=\bar{y}-\beta_{1}^{p} \bar{x}$ and

$$
\beta_{1}^{p}=\frac{\left(S_{y y}-S_{x x}\right) \pm \sqrt{\left(S_{y y}-S_{x x}\right)^{2}+4 S_{x y}^{2}}}{2 S_{x y}},
$$

(provided $S_{x y} \neq 0$ ).

Note with $S_{x y} \neq 0$ and $S_{x x}=S_{y y}$, then $\beta_{1}^{p}= \pm 1$ showing that under standardization this method is functionally independent of the correlation between $x$ and $y$ ! 
For squared geometric mean errors, we minimize $S S E_{g}\left(\beta_{0}, \beta_{1}\right)$ $=\sum_{i=1}^{n}\left(\sqrt{\left|a_{i} b_{i}\right|}\right)^{2}=\sum_{i=1}^{n} a_{i}^{2} /\left|\beta_{1}\right|$ with solutions $\beta_{0}^{g}=\bar{y}-\beta_{1}^{g} \bar{x}$ and $\beta_{1}^{g}= \pm \sqrt{S_{y y} / S_{x x}}$. Note that $\beta_{1}^{g}$ is always functionally independent of the correlation between $x$ and $y$ and also under standardization $b_{1}^{g}= \pm 1$ as in the perpendicular model.

The solutions to the above equations for both $\beta_{1}^{p}$ and $\beta_{1}^{g}$ are also roots of $P_{4}\left(\beta_{1}\right)$ for particular values of $\lambda$ which can be seen from the geometry of the model. See [3] and [2] for applications of the perpendicular and geometric mean estimators.

\section{Minimizing Squared Weighted Average Errors}

If the user wishes to incorporate the effect of different variances in $x$ and $y$, this can be achieved by using a weighed average of the squared vertical and squared horizontal errors with $(0 \leq \alpha \leq 1)$ and $S S E_{w}=\alpha S S E_{v}+(1-\alpha) S S E_{h}$. A typical value for $\alpha$ might be $\alpha=\sigma_{y}^{2} /\left(\sigma_{x}^{2}+\sigma_{y}^{2}\right)$ to standardize the data. Recall from Section 2 that $S S E_{o}=\lambda^{2} S S E_{v}+(1-\lambda)^{2} S S E_{h}$. On setting $(1-\lambda)^{2} / \lambda^{2}=(1-\alpha) / \alpha$, we get the quadratic equation $(2 \alpha-1) \lambda^{2}-2 \alpha \lambda+\alpha=0$, which has root

$$
\lambda= \begin{cases}\frac{\alpha-\sqrt{\alpha(1-\alpha)}}{(2 \alpha-1)} & \alpha \neq \frac{1}{2} \\ \frac{1}{2} & \alpha=\frac{1}{2} .\end{cases}
$$

\section{Case Study}

In this section, we introduce the median estimator $\beta_{1}^{m}$ using $P_{4}\left(\beta_{1}\right)$ with $\lambda=1 / 2$. Our small case study reveals the desirable robustness inherent in the median estimator. The data set is from [2] with $n=40$. The case study shows that the perpendicular estimator is highly influenced by outliers in the data, with the vertical and horizontal estimators also being significantly influenced by outliers. The geometric mean estimator, as expected, is more robust; and our median estimator, introduced in this paper, being the most robust in this case study. For the Weighted Average procedure, $\alpha=S_{y y} /\left(S_{y y}+S_{x x}\right)=0.671$ which from Equation 4 yields $\lambda=0.588$. 
The first table below gives the values for the slope $\beta_{1}, y$-intercept $\beta_{0}, \lambda$, and $S S E$. To study the effect of outliers, we pick a row from the data set and perturb the values by some factor.

The second table contains the basic values and, in addition, the square of the shifts in the slope and $y$-intercept caused by perturbing the $x$-data by a factor of 7.5 for the data point for case $k=5$. Note that the median estimator has the smallest squared shift distance. The third table shows similar values by perturbing the $y$-data by a factor of 0.5 for case $k=5$. Note that the perpendicular model has been greatly influenced by this one outlier.

\begin{tabular}{lllllll} 
& Vert & Horiz & Perp & Geom & Median & Wt Avg \\
\hline \hline$\beta_{1}$ & 1.28 & 1.59 & 1.48 & 1.43 & 1.38 & 1.35 \\
$\beta_{0}$ & 136 & 104 & 115 & 121 & 126 & 130 \\
$\lambda$ & 1.00 & 0.00 & 0.312 & 0.412 & 0.500 & 0.588 \\
$S S E$ & 12565 & 6163 & 4330 & 4494 & 4908 & 5581
\end{tabular}

Table 1. Gill Data for Vertical (Vert), Horizontal (Horiz), Perpendicular (Perp), Geometric Mean (Geom), Median and Weighted Average (Wt Avg) Procedures

\begin{tabular}{llllll} 
& Vert & Horiz & Perp & Geom & Median \\
\hline \hline$\beta_{1}$ & 0.0937 & 2.33 & 0.118 & 0.467 & 0.654 \\
$\beta_{0}$ & 259 & -4.39 & 256 & 215 & 193 \\
$S S E$ & 62007 & 284364 & 61327 & 95987 & 129360 \\
$\left(\beta_{1}^{*}-\beta_{1}\right)^{2}$ & 1.41 & 0.541 & 1.87 & 0.923 & 0.531 \\
$\left(\beta_{0}^{*}-\beta_{0}\right)^{2}$ & 15040 & 11723 & 19855 & 8818 & 4506
\end{tabular}

Table 2. Gill Data perturbed with $x^{*}[5]=7.5 x[5]$

\begin{tabular}{llllll} 
& Vert & Horiz & Perp & Geom & Median \\
\hline \hline$\beta_{1}$ & 0.875 & 1.99 & 1.51 & 1.32 & 1.23 \\
$\beta_{0}$ & 174 & 57.2 & 107 & 127 & 137 \\
$S S E$ & 30977 & 17770 & 13339 & 13841 & 14521 \\
$\left(\beta_{1}^{*}-\beta_{1}\right)^{2}$ & 0.165 & 0.161 & 0.000717 & 0.0116 & 0.0228 \\
$\left(\beta_{0}^{*}-\beta_{0}\right)^{2}$ & 1410 & 4875 & 3446 & 2789 & 2312
\end{tabular}

Table 3. Gill Data perturbed with $y^{*}[4]=0.5 y[4]$ 
We replicated the above perturbation procedure for each of the $n=40$ cases and record in Table 4 and Table 5 the average squared change in slope and the average squared change in the $y$-intercept denoted $\left\{E\left(\beta_{1}^{*}-\beta_{1}\right)^{2}, E\left(\beta_{0}^{*}-\beta_{0}\right)^{2}\right\}$ by perturbing the original $x$ data and $y$-data values by a factor of $\{7.5,0.5\}$ respectively. Table 6 records the average squared changes where the data has been jointly perturbed for $(x[k], y[k])$ by the factors $\{7.5,0.5\}$ respectively.

\begin{tabular}{llllll} 
& Vert & Horiz & Perp & Geom & Median \\
\hline$E\left(\beta_{1}^{*}-\beta_{1}\right)^{2}$ & 1.41 & 4.01 & 1.87 & 1.07 & 0.656 \\
$E\left(\beta_{0}^{*}-\beta_{0}\right)^{2}$ & 14966 & 57192 & 19820 & 10358 & 5649
\end{tabular}

Table 4. Gill Data perturbed with $x^{*}[k]=7.5 x[k]$

\begin{tabular}{llllll} 
& Vert & Horiz & Perp & Geom & Median \\
\hline \hline$E\left(\beta_{1}^{*}-\beta_{1}\right)^{2}$ & 0.0163 & 0.201 & 0.0968 & 0.0333 & 0.0143 \\
$E\left(\beta_{0}^{*}-\beta_{0}\right)^{2}$ & 165 & 2509 & 1276 & 488 & 229
\end{tabular}

Table 5. Gill Data perturbed with $y^{*}[k]=0.5 y[k]$

\begin{tabular}{llllll} 
& Vert & Horiz & Perp & Geom & Median \\
\hline$E\left(\beta_{1}^{*}-\beta_{1}\right)^{2}$ & 1.90 & 20.6 & 2.59 & 0.975 & 0.487 \\
$E\left(\beta_{0}^{*}-\beta_{0}\right)^{2}$ & 20175 & 258272 & 27880 & 8611 & 3364
\end{tabular}

Table 6. Gill Data perturbed with $\left\{x^{*}[k]=7.5 x[k], y^{*}[k]=0.5 y[k]\right\}$

The results in Table 4 with an outlier in the $x$-data show the sensitivity with the vertical, horizontal and perpendicular procedures. The results in Table 5 with an outlier in the $y$-data show the sensitivity with the horizontal and perpendicular procedures. Table 6 , with $(x, y)$ both perturbed, shows the robustness of the geometric and median procedures with the median estimators uniformly superior to the geometric estimators in this small case study. These preliminary results commend the method for further investigation. 


\section{REFERENCES}

[1] R. J. Adcock, A problem in least-squares, The Analyst, 5 (1878), 53-54.

[2] J. L. Gill, Biases in regression when prediction is inverse to causation, American Society of Animal Science 64 (1987), 594-600.

[3] L. Leng, T. Zhang, L. Kleinman, and W. Zhu, Ordinary least square regression, orthogonal regression, geometric mean regression and their applications in aerosol science, Journal of Physics: Conference Series 78 (2007), 012084012088.

[4] L. Yang, Recent advances on determining the number of real roots of parametric polynomials, J. Symbolic Computation 28 (1999), 225-242.

Diarmuid O'Driscoll,

Department of Mathematics and Computer Studies,

Mary Immaculate College,

Limerick, Ireland,

diarmuid.odriscoll@mic.ul.ie

Donald E. Ramirez,

Department of Mathematics,

University of Virginia,

Charlottesville, VA 22904, USA

der@virginia.edu

Rebecca Schmitz,

ITCEP,

University of Minnesota,

Minneapolis, MN 55455, USA

schm2676@umn.edu

Received on 2 October 2008. 